\title{
Impact of obstructive sleep apnea complicated with type 2 diabetes on long- term cardiovascular risks and all-cause mortality in elderly patients
}

Xiaofeng Su${ }^{1+}$, Jian Hua $\mathrm{Li}^{2+}$, Yinghui Gao ${ }^{3+}$, Kaibing Chen ${ }^{4}$, Yan Gao ${ }^{5}$, Jing Jing Guo ${ }^{6}$, Min Shi ${ }^{7}$, Xiao Zou ${ }^{2}$,

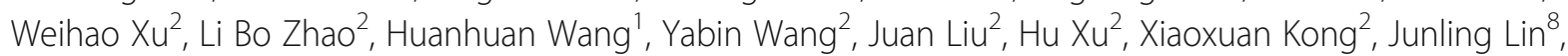
Xiaoshun Qian ${ }^{7^{*}}$, Jiming $\mathrm{Han}^{{ }^{1 *}}$ and Lin Liu ${ }^{7^{*}}$

\begin{abstract}
Background: The prognostic significance of obstructive sleep apnea (OSA) in elderly patients with type 2 diabetes is unclear. The aim of this study was to determine the risk of cardiovascular disease (CVD) and mortality in elderly patients with OSA complicated with type 2 diabetes compared to patients with OSA without type 2 diabetes.

Methods: From January 2015 to October 2017, 1113 eligible elderly patients with OSA, no history of cardiovascular, $\geq 60$ years of age, and complete follow-up records were enrolled in this consecutive multicentre prospective cohort study. All patients had completed polysomnography (PSG) examinations. An apnoea-hypopnoea index of $\geq 5$ events per hour recorded by polysomnography was defined as the diagnostic criterion for OSA. We collected baseline demographics, clinical characteristics, sleep parameters and follow-up outcomes. The primary aim of this study was to identify the risk of incident major adverse cardiovascular events (MACE). Secondary outcomes were all-cause mortality, components of MACE and a composite of all events. Kaplan-Meier survival analysis and Cox proportional hazards models were used to evaluate whether type 2 diabetes was associated with incident events.
\end{abstract}

\footnotetext{
*Correspondence: qianxs@yahoo.com; yadxhjm@163.com; liulin715@qq.com

${ }^{+}$Xiaofeng Su, Jian Hua Li and Yinghui Gao contributed equally to this work.

${ }^{7}$ Department of Respiratory and Critical Care Medicine of the Second

Medical Center \& National Clinical Research Center for Geriatric Diseases,

Chinese PLA General Hospital, 28 Fuxing Road, Beijing, China

${ }^{1}$ Medical College, Yan'an University, Yan'an, Shanxi Province, China

Full list of author information is available at the end of the article
}

\section{$\triangle B M C$}

(c) The Author(s). 2021 Open Access This article is licensed under a Creative Commons Attribution 4.0 International License, which permits use, sharing, adaptation, distribution and reproduction in any medium or format, as long as you give appropriate credit to the original author(s) and the source, provide a link to the Creative Commons licence, and indicate if changes were made. The images or other third party material in this article are included in the article's Creative Commons licence, unless indicated otherwise in a credit line to the material. If material is not included in the article's Creative Commons licence and your intended use is not permitted by statutory regulation or exceeds the permitted use, you will need to obtain permission directly from the copyright holder. To view a copy of this licence, visit http://creativecommons.org/licenses/by/4.0/ The Creative Commons Public Domain Dedication waiver (http://creativecommons.org/publicdomain/zero/1.0/) applies to the data made available in this article, unless otherwise stated in a credit line to the data. 
Results: A total of 266 (23.9\%) patients had OSA complicated with type 2 diabetes. MACE occurred in 97 patients during the median 42-month follow-up. Kaplan-Meier survival curves indicated a significant relationship between type 2 diabetes and MACE (log-rank $P=0.003$ ). Multivariable Cox regression analysis showed that type 2 diabetes increased the risk of MACE ( $H R=1.64,95 \% \mathrm{Cl}: 1.08-2.47, P=0.019)$, hospitalisation for unstable angina $(H R=2.11$, 95\% Cl:1.23-3.64, $P=0.007)$ and a composite of all events in elderly patients with OSA (HR=1.70, 95\% Cl:1.17-2.49, $P=0.007)$. However, there were no significant differences in the incidence of cardiovascular death, all-cause mortality, $\mathrm{Ml}$ and hospitalisation for heart failure between patients with and without diabetes $(P>0.05)$. The subgroup analysis demonstrated that females (AHR $=2.46,95 \% \mathrm{Cl}: 1.17-5.19, P=0.018), \geq 70$ years (AHR $=1.95,95 \%$ Cl:1.08-3.52, $P=0.027)$, overweight and obese (AHR $=2.04,95 \% \mathrm{Cl}: 1.29-3.33, P=0.002)$ with mild OSA (AHR $=2.42$, 95\% Cl: 1.03-5.71, $P=0.044$ ) were at a higher risk for MACE by diabetes.

Conclusion: OSA and type 2 diabetes are interrelated and synergistic with MACE, hospitalisation for unstable angina and a composite of all events development. Overweight and obese females, $\geq 70$ years with mild OSA combined with type 2 diabetes presented a significantly high MACE risk.

Keywords: Obstructive sleep apnea, Type 2 diabetes, Elderly, Major adverse cardiovascular events, Mortality, Cardiovascular disease

\section{Background}

OSA is a chronic and fatal sleep disorder, and OSArelated CVD and mortality worsen the quality of life in patients with OSA [1]. Some studies show that OSA is associated with an increased risk of death and cardiovascular disease $[2,3]$. Type 2 diabetes is a frequent comorbidity in patients with OSA [4-6]. Intermittent hypoxaemia and sleep fragmentation in OSA could contribute independently to the development of insulin resistance, glucose intolerance and type 2 diabetes. Conversely, type 2 diabetes may increase predisposition to, or accelerate the progression of OSA, possibly through the development of peripheral neuropathy and abnormalities of ventilatory and upper airway neural control $[7,8]$. The prevalence of OSA in patients with type 2 diabetes ranges from 50 to $80 \%$ $[9,10]$. A cross-sectional study confirmed an association between OSA and type 2 diabetes [11]. A longitudinal study showed that OSA patients with type 2 diabetes have higher CVD mortality [12], while another study revealed that people with type 2 diabetes do not seem to have an increased risk of death and myocardial infarction than the general population [13]. Some researchers found that respiratory sleep disorders in the young, middle-aged patients were independent of atrial fibrillation, but there was no association in elderly patients [14]. From a physiological perspective, the elderly have greater hypoxic tolerance, and repeated intermittent hypoxia protects the myocardium against ischaemic injury [15]. A previous study showed that the cardiovascular disease mortality rate for OSA patients younger than 50 years was higher, but the risk significantly reduced after 50 years [16]. Other researchers confirmed that OSA in elderly patients was not related to the increased risk of cardiovascular disease [17]. Thus, the posed question was whether type 2 diabetes complications increase the risk of CVD, all-cause mortality and a composite of all events in patients with OSA, especially in the elderly OSA population.

Therefore, we investigated the association between type 2 diabetes and the incidence of MACE, all-cause mortality and a composite of all events in patients with OSA using the multicentre population-based prospective cohort data.

\section{Methods \\ Study design and participants}

The study is a large-scale, multi-center, prospective, cohort study to assess the association of type 2 diabetes with cardiovascular risks and all-cause mortality in elderly patients with OSA. We Consecutive enrolled 1290 elderly patients with OSA from January 2015 to October 2017. All patients were hospitalized at the sleep medicine center of six hospitals, respectively, which including Chinese PLA General Hospital, Peking University International Hospital, Peking University People's Hospital, Beijing Chaoyang Hospital, 960th Hospital of PLA, and the affiliated Hospital of Gansu University of Chinese Medicine. Patients with OSA, no history of myocardial infarction (MI) or hospitalisation for unstable angina or heart failure, aged $\geq 60$ years, and had completed polysomnography (PSG) examinations were eligible for inclusion. OSA was defined as an apnea-hypopnea index of $\geq 5$ events per hr. The AHI was defined as the number of apnoea and hypopnea per hour of sleep. We excluded 177 patients based on the following criteria: (1) diagnosis of type 1 diabetes between 2015 and $2017(n=48)$; (2) one standard treatment of continuous positive airway pressure (CPAP) for OSA $(n=71)$; (3) previous history 
of myocardial infarction (MI), hospitalisation for unstable angina or heart failure $(n=34)$; (4) presence of malignant tumours $(n=3)$; (5) presence of mental disorders $(n=4)$. Furthermore, we excluded those lost during the follow-up ( $n=17)$; the final study subjects were 1113 aging patients with OSA. This study conformed to the STROBE (Strengthening the Reporting of Observational studies in Epidemiology) guidelines and was carried out in accordance with the Declaration of Helsinki. The Ethics Committee of PLA General Hospital (S2019-352-01) approved the study. Written informed consent was available from all participants.

\section{Polysomnography (PSG)}

All patients underwent an overnight sleep monitoring (from 21: 00 to 7: 00 the next day) after clinical stabilization during hospitalization at sleep centre (within 1 weeks after admission) and sleep parameters were recorded using portable laboratory-based polysomnography (PSG) instrument (Compumedics, Melbourne, Australia), as described previously [18]. Sleep parameters from PSG were as follows: continuous polygraphic recording from surface leads for electroencephalography, electrooculography, electromyography, electrocardiography, thermistors for nasal and oral airflow, thoracic and abdominal impedance belts for respiratory effort, pulse oximetry for oxyhaemoglobin concentration, tracheal microphone for snoring and a sensor for the position during sleep. Analysis of sleep tests was scored according to the American Academy of Sleep Medicine 2017 guidelines [19]. Patients PSG records of each hospital were automatically analysed and manually calibrated by manually twice (by two professional sleep technologist), both of whom were blinded to the demographic and clinical characteristics. Further analysis was performed in cases of discrepancy by a senior physician in sleep medicine. OSA was defined as AHI $\geq 5$ events/hour. Patients with AHI $<5$ events/hour were considered as the non- OSA. Patients with OSA (AHI $\geq 5$ ), particularly those with excessive daytime sleepiness, were referred to the sleep center for further evaluation. An apnea was defined as the continuous cessation of airflow for more than $10 \mathrm{~s}$, whereas a hypopnea was defined as a reduction in airflow by $50 \%$ with a duration of at least $10 \mathrm{~s}$ or a reduction of airflow or respiratory effort by $30 \%$ for more than $10 \mathrm{~s}$, accompanied by an electroencephalographic arousal, a $4 \%$ or greater oxygen desaturation, or both. The AHI was defined as the number of apnoea and hypopnoea per hour of sleep. The oxygen desaturation index (ODI) was defined as a $\mathrm{SaO} 2$ drop of $\geq 3 \%$. OSA was classified as mild (AHI of 5 to 14.9), moderate (AHI of 15 to 30$)$ or severe $(\mathrm{AHI}>30)[19,20]$.

\section{Covariates}

The following potential confounders and risk factors were extracted from clinical data: age, sex, body mass index (BMI), blood pressure (BP), plasma glucose, HbA1c and self-reported smoking and alcohol use. Sleep parameters were as follows: AHI, ODI, mean oxygen saturation and lowest oxygen saturation. Comorbidities were identified at baseline (carotid atherosclerosis, hyperlipidaemia, atrial fibrillation, hypertension [HTN], chronic obstructive pulmonary disease [COPD], coronary heart disease [CHD] and diabetes from the hospital administrative database over a six-month period before the diagnostic sleep study. These data were assessed by an interviewer who administered the Unified epidemiological questionnaire and were reviewed by three physicians. Blood was drawn for biochemical analysis after overnight fasting. Plasma glucose, $2 \mathrm{~h}$ glucose concentration in the standard $75 \mathrm{~g}$ oral glucose tolerance test were measured using a Roche C8000 Automatic Analyzer. The categories of covariates were listed in Supplementary Table s- 1 .

\section{Definitions}

Body mass index (BMI) was calculated as weight $(\mathrm{kg}) \mathrm{di}$ vided by height $\left(\mathrm{m}^{2}\right)$. Current smoking was defined as at least one cigarette a day, and current drinking was defined as drinking once per week for at least half a year. Systolic blood pressure (SBP) and diastolic blood pressure (DBP) were measured three times. Hypertension was defined present if the mean of at least two consecutive measurements of SBP/DBP $\geq 140 / 90 \mathrm{mmHg}$ or the use of antihypertension medication [21]. Dyslipidemia using Chinese Guideline for the management of hyperlipidemia in adults was defined as (1) serum cholesterol levels $\geq 4.7 \mathrm{mmol} / \mathrm{L}$, (2) triglyceride levels $\geq 2.3 \mathrm{mmol} / \mathrm{L}$, or (3) low-density lipoprotein levels $\geq 4.1 \mathrm{mmol} / \mathrm{L}$. Patients who meet one of these three items are defined as having hyperlipidemia [22]. Atrial fibrillation was defined based on the ESC 2016 guidelines [23]. Carotid atherosclerosis, coronary heart disease (CHD) and chronic obstructive pulmonary disease (COPD) were determined by a record of a relevant diagnostic clinical (Read) code indicating the presence of the condition [24].

\section{Diagnostic criteria}

We considered any of the following parameters for a diagnosis of type 2 diabetes: (1) diabetes symptoms (typical symptoms, including polydipsia, polyuria and unexplained weight loss) and plasma glucose $\geq 11.1 \mathrm{mmol} / \mathrm{L}$ $(200 \mathrm{~g} / \mathrm{L})$ at any time; (2) fasting plasma glucose $\geq 7.0$ $\mathrm{mmol} / \mathrm{L}(126 \mathrm{~g} / \mathrm{L})$; (3) OGTT2h plasma glucose $\geq 11.1$ $\mathrm{mmol} / \mathrm{L}(200 \mathrm{~g} / \mathrm{L})$ [25]. 


\section{Treatment and management}

The subjects with OSA were split into diabetes group $(n=266)$ and non-diabetes group $(n=847)$. All patients in diabetes group treated by taking diabetes medications. And 1113 patients received standard care during OSA hospitalization at the sleep medicine center of six hospitals according to current guidelines [19]. Patients with $\mathrm{AHI}<5$ events/hour were seen as the non- OSA. Patients with OSA (AHI $\geq 5)$, particularly those with excessive daytime sleepiness, were referred to the sleep center for further evaluation.

\section{Follow-up}

One thousand one hundred thirteen patients with OSA were followed up from the diagnosed time of PSG assessment to December 2020 for MACE, cardiovascular death, all-cause mortality, MI, hospitalisation for unstable angina or heart failure and the development of composite of all events and was performed at 1 month, 3 months, 6 months, 1 year, and then every 6 months thereafter (at least 3 months and up to 1 years). The participants' outcomes were collected by a clinic visit, medical chart review, or telephone calls by two investigators who were blinded to patients' PSG results every 6 months. All clinical events were confirmed by source documentation and were adjudicated by the clinical event committee. For the current study, all patients received standard health care depending on their disease status during a median follow-up of 42 months. The primary outcome was MACE, including cardiovascular death, MI and hospitalisation for unstable angina or heart failure. Secondary outcomes were all-cause mortality, individual components of MACE and a composite of all events. The study ended if patients followed up the incidence of new-onset major adverse cardiovascular events (MACE) or all-cause mortality, which was the first MACE or all-cause mortality event for that patient. Two or more MACE or all-cause mortal event were uniformly counted as one event, with the first event time and event reported as the outcome.

\section{Statistical analysis}

Demographics, clinical characteristics and sleep parameters in the study subjects were categorised according to type 2 diabetes using a Pearson's Chi-square test and an independent t-test. Data were indicated as percentages for categorical variables or mean \pm standard deviation for normally distributed continuous variables. Skewed variables were presented as median (interquartile range) and compared using the Mann-Whitney $U$ test. Crude and adjusted hazard ratios (AHR) and their corresponding 95\% confidence intervals (CI) for the association between type 2 diabetes and incidence of all events were calculated using Cox proportional hazards regression models. Kaplan-Meier curves were used to visualise the association between type 2 diabetes and adverse events. Two Cox proportional hazards regression models were conducted to examine the association between type 2 diabetes coexisting with OSA and long-term cardiovascular risks and all-cause mortality. Model 1, unadjusted analysis; Model 2(adjusted analysis), Model 1 plus sex, BMI, plasma glucose, alcohol use, HbA1c, WHR, waist circumference, comorbidities of CHD, hyperlipidemia, hypertension, carotid atherosclerosis, atrial fibrillation, and sleep parameters of ODI, TST, T90, TSA90, WHR, waist circumference, AHI, average apnea time and maximum apnea time. All analyses were conducted using the SPSS statistical software (version 25.0, SPSS Inc., Chicago, Illinois, USA).

\section{Results}

\section{Baseline characteristics}

In total, 1290 consecutive eligible elderly patients with OSA were prospectively enrolled, all of whom underwent a successful overnight sleep study. After exclusion of patients according to predefined criteria, 1113 study subjects with OSA aged $\geq 60$ years in the final analysis (Fig. 1); 285 patients (25.6\%) had mild OSA, 336 (30.2\%) had moderate OSA, 492 (44.2\%) had severe OSA and 266 (23.9\%) had OSA complicated with type 2 diabetes. Diabetes patients had higher severe OSA rates than nondiabetes patients $(P=0.011)$. Table 1 present the general characteristics of study participants according to type 2 diabetes. The proportions of alcohol use (13.2\% vs. $7.5 \%$ ), comorbidities (CHD) [39.8\% vs. 16.5\%], hyperlipidaemia $[47.7 \%$ vs. $21.8 \%]$, hypertension $[81.6 \%$ vs. $57.7 \%]$, atrial fibrillation [11.6\% vs. $4.5 \%]$ and carotid atherosclerosis [37.2\% vs. $22.1 \%]$ ) in individuals with diabetes were significantly higher than in those without diabetes in patients with OSA. Patients with diabetes also showed significantly higher levels of average systolic (140 mmHg vs. $130 \mathrm{mmHg}$ ) and diastolic $(80 \mathrm{mmHg}$ vs. $76 \mathrm{mmHg}) \mathrm{BP}$, plasma glucose $(6.47 \mathrm{mmol} / \mathrm{L}$ vs. 5.18 $\mathrm{mmol} / \mathrm{L}$ ), age (67 year vs. 65 year), waist circumference (91 mm vs. $89 \mathrm{~mm})$, WHR (0.92 vs. 0.87), average apnea time $(22.72 \mathrm{~s}$ vs. $21.91 \mathrm{~s})$, AHI (30.3 times/h vs. 25.4 times/h), BMI $\left(27.27 \mathrm{~kg} / \mathrm{m}^{2}\right.$ vs. $\left.25.95 \mathrm{~kg} / \mathrm{m}^{2}\right)$ and HbA1c (38.44 $\mathrm{mmol} / \mathrm{mol}$ vs. $36.80 \mathrm{mmol} / \mathrm{mol})$.

\section{Impact of type $\mathbf{2}$ diabetes on adverse events during follow up \\ Primary outcome: MACE}

Crude numbers of adverse events are shown in Table 2. This study examined 97 events of MACE (8.7\%) during a median follow-up period of 42 months (range 1 to 72 months): 33 (12.4\%) in diabetes patients and 64 (7.6\%) in non-diabetes patients. Kaplan-Meier analysis showed that the cumulative event rate of MACE among OSA 


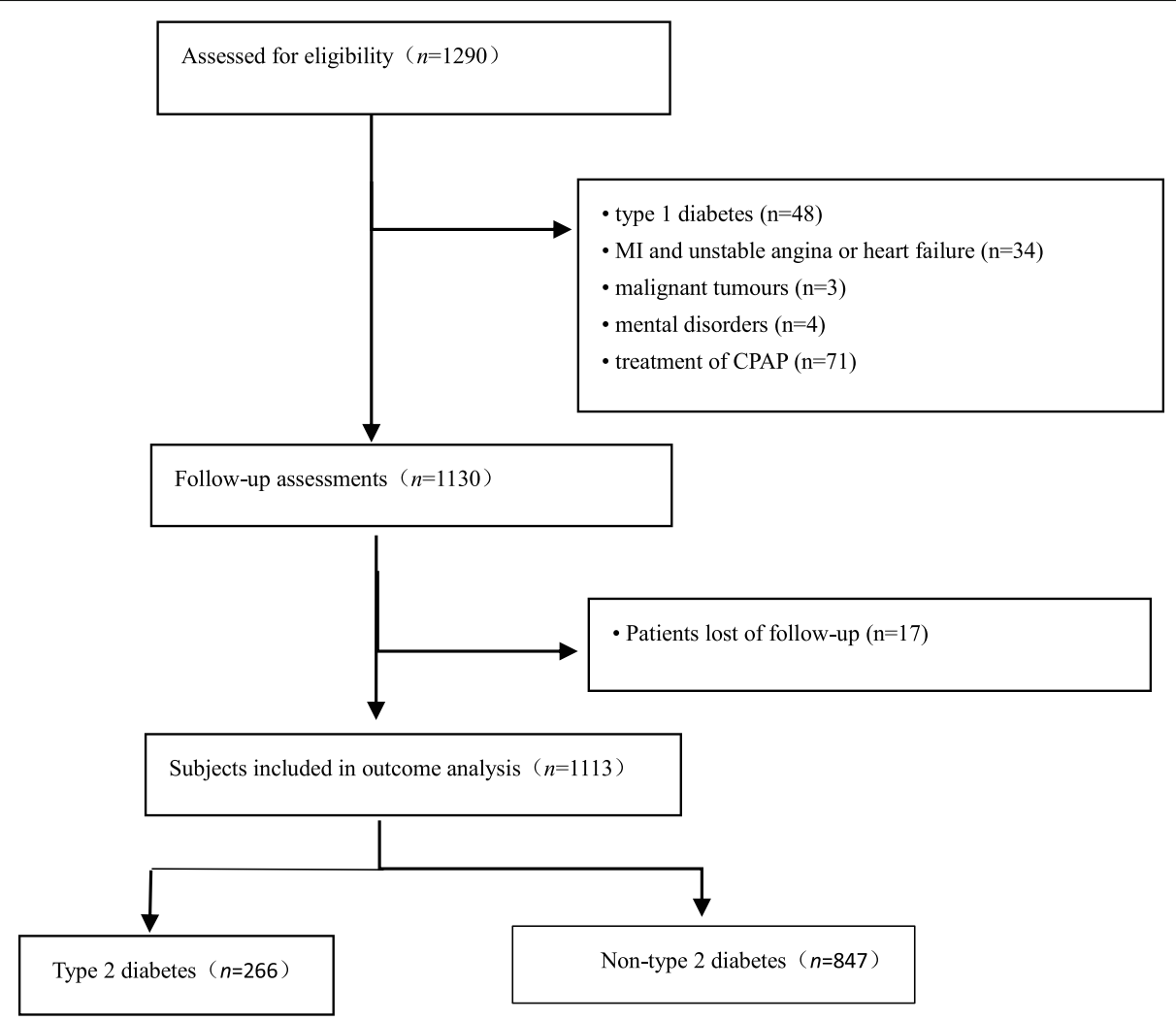

Fig. 1 Study flowchart. OSA, obstructive sleep apnea; CPAP indicates continuous positive airway pressure

patients with type 2 diabetes was significantly higher than in OSA patients without type 2 diabetes (Log-rank test: $P=0.003$ ) (Fig. 2). Table 3 showed unadjusted and adjusted HRs for incidence of MACE according to diabetes with OSA. Following adjustment for sex, BMI, plasma glucose, alcohol use, HbA1c, ODI, TST, T90, TSA90, WHR, waist circumference, AHI, average apnea time, maximum apnea time, and comorbidities of CHD, hyperlipidaemia, hypertension, carotid atherosclerosis, atrial fibrillation and diabetes significantly increased the risk of MACE ( $\mathrm{HR}=1.64$, 95\% CI:1.08-2.47, $P=0.019)$ in elderly patients with OSA. In the subgroup analysis, adjusted hazard ratios for MACE by diabetes were higher in overweight and obese females $\geq 70$ years and patients with mild OSA (Table 4).

\section{Secondary outcomes: all-cause mortality, components of $M A C E$, and a composite of all events}

Forty-three patients died during the follow-up period, the proportions of diabetes group vs. non-diabetes group (5.6\% vs. 3.3\%), Table 2. The univariate analysis showed that diabetes was associated with a higher (approximately 4-year) risk of all-cause mortality in elderly patients with OSA ( $\mathrm{HR}=2.02,95 \% \mathrm{CI}: 1.07-3.80, P=0.029)$. However, for adjusted hazard ratios for all-cause mortality, the trend of increased risk was statistically insignificant $(\mathrm{HR}=1.54$,
95\% CI: $0.82-2.90, P=0.187)$, Table 3 . In the adjusted Cox regression analysis, there were no significant differences in the incidence of cardiovascular death, MI and hospitalisation for heart failure between patients with and without diabetes $(P>0.05)$, Table 3 . However, multivariable Cox regression analyses showed that diabetes significantly increased the risk of a composite of all events $(\mathrm{HR}=1.70,95 \% \mathrm{CI}: 1.17-2.49, P=0.007)$ and hospitalisation for unstable angina $(\mathrm{HR}=2.11,95 \% \mathrm{CI}: 1.23-3.64$, $P=0.007)$, Table 3. Kaplan-Meier curves were used to present the relationship between the two events and diabetes for a different view (Log-rank test: $P=0.000, P=$ 0.026, respectively,), Figs. 3, 4 .

\section{Treatment and management}

Patients in diabetes group treated by taking diabetes medications. A total of 1113 patients included, standard OSA treatment (CPAP) has been excluded, and patients with other therapy of OSA, including surgery $(n=49)$, weight loss $(n=142)$ and oral appliance therapy $(n=54)$ were included in our study. We have compared the impact of these therapy methods on the primary outcome (MACE) in supplementary Table S-2. In both groups, our study founded that patients undergone oral appliance therapy for OSA fell short of statistical significance for the risk of MACE compared to patients of OSA 
Table 1 General characteristics of study subjects according to type 2 diabetes

\begin{tabular}{|c|c|c|c|c|}
\hline & $\begin{array}{l}\text { Total } \\
(n=1113)\end{array}$ & $\begin{array}{l}\text { Diabetes } \\
(n=266)\end{array}$ & Non-diabetes $(n=847)$ & $P$-Value \\
\hline \multicolumn{5}{|l|}{ Demographics } \\
\hline Age, y & $66.0(62.0,71.0)$ & $67(64.0,72.0)$ & $65.0(62.0,70.0)$ & 0.000 \\
\hline Male, n (\%) & $675(60.6)$ & $171(64.3)$ & $504(59.5)$ & 0.164 \\
\hline $\mathrm{BMI}, \mathrm{kg} / \mathrm{m}^{2}$ & $26.30(23.88,28.80)$ & $27.27(24.50,29.80)$ & $25.95(23.63,28.31)$ & 0.000 \\
\hline Waist circumference, cm & $90(80,99)$ & $91(80,101)$ & $89(80,99)$ & 0.010 \\
\hline Neck circumference, cm & $39(35,41)$ & $38(36,40)$ & $38(35,40)$ & 0.653 \\
\hline WHR & $0.88(0.78,1.01)$ & $0.92(0.79,1.05)$ & $0.87(0.78,0.98)$ & 0.019 \\
\hline $\mathrm{SBP}, \mathrm{mmHg}$ & $130(122,143)$ & $140(130,160)$ & $130(120,140)$ & 0.000 \\
\hline $\mathrm{DBP}, \mathrm{mmHg}$ & $76(70,83)$ & $80(70,87)$ & $76(70,82)$ & 0.003 \\
\hline Smoking, n (\%) & $160(14.4)$ & $31(11.7)$ & $129(15.2)$ & 0.252 \\
\hline Drinking, n (\%) & $98(8.8)$ & $35(13.2)$ & $63(7.5)$ & 0.010 \\
\hline Plasma glucose, mmol/L & $6.18(5.38,6.19)$ & $6.47(6.04,7.91)$ & $5.18(4.57,5.90)$ & 0.048 \\
\hline $\mathrm{HbA} 1 \mathrm{c}, \%$ & $5.52(5.10,5.63)$ & $5.67(5.44,6.43)$ & $4.32(4.04,5.62)$ & 0.045 \\
\hline $\mathrm{HbA} 1 \mathrm{c}, \mathrm{mmol} / \mathrm{mol}$ & $36.81(32.23,36,9)$ & $38.44(36.10,46.70)$ & $36.80(31.61,37.83)$ & 0.043 \\
\hline \multicolumn{5}{|l|}{ Sleep parameters } \\
\hline AHI, events/h & $26.7(14.6,45.2)$ & $30.3(17.2,48.7)$ & $25.4(14.0,44.3)$ & 0.010 \\
\hline ODI, events/h & $21.4(10.2,40.5)$ & $22.9(10.9,41.5)$ & $20.7(10.1,39.8)$ & 0.467 \\
\hline $\mathrm{MSpO}_{2}, \%$ & $93(92,95)$ & $94(92,95)$ & $93(92,95)$ & 0.184 \\
\hline $\mathrm{LSpO}_{2}, \%$ & $80(72,85)$ & $80(70,86)$ & $81(73,85)$ & 0.367 \\
\hline TST, h & $7.03(6.11,7.42)$ & $7.09(6.16,7.43)$ & $7.01(5.96,7,42)$ & 0.139 \\
\hline TSA90, min & $14.11(2.28,60.32)$ & $14.19(2.48,60.93)$ & $12.73(1.98,56.81)$ & 0.179 \\
\hline T90, \% & $3.51(0.61,15.37)$ & $3.51(0.64,15.81)$ & $3.29(0.49,14.49)$ & 0.272 \\
\hline Maximum apnea time, $s$ & $63.12(42.44,85.20)$ & $63.51(41.96,86.13)$ & $61.80(42.11,84.23)$ & 0.722 \\
\hline Average apnea time, s & $22.47(19.46,25.46)$ & $22.72(19.46,25.68)$ & $21.91(19.46,25.10)$ & 0.095 \\
\hline \multicolumn{5}{|l|}{ Medical history, n (\%) } \\
\hline Severity of OSA & & & & 0.030 \\
\hline Mild OSA & $285(25.6)$ & $55(20.7)$ & $230(27.2)$ & \\
\hline Moderate OSA & $336(30.2)$ & $76(28.6)$ & $260(30.7)$ & \\
\hline Severe OSA & $492(44.2)$ & $135(50.8)$ & $357(42.1)$ & \\
\hline $\mathrm{CHD}$ & $246(22.1)$ & $106(39.8)$ & $140(16.5)$ & 0.000 \\
\hline Hyperlipidemia & $312(28.0)$ & $128(47.7)$ & $185(21.8)$ & 0.000 \\
\hline Hypertension & $706(63.4)$ & 217 (81.6) & $489(57.7)$ & 0.000 \\
\hline Atrial fibrillation & $69(6.2)$ & 31 (11.6) & $38(4.5)$ & 0.000 \\
\hline Carotid atherosclerosis & $286(25.7)$ & 99 (37.2) & $187(22.1)$ & 0.000 \\
\hline COPD & $78(7.0)$ & $22(8.3)$ & $56(6.6)$ & 0.355 \\
\hline
\end{tabular}

$B M I$ body mass index, WHR waist/hip ratio, $S B P$ systolic blood pressure, $D B P$ diastolic blood pressure, $A H I$ the apnea-hypopnea index, $O D I$ the oxygen desaturation index, MSpO2 the mean pulse oxygen saturation, $L S p O 2$ the lowest pulse oxygen saturation, TSA90 the duration of time with $\mathrm{SaO}<209 \%$, T90 percentage of the times for $\mathrm{SaO}_{2}<90 \%$ in total monitoring time during overnight sleep, OSA obstructive sleep apnea, CHD coronary heart disease, COPD chronic obstructive pulmonary disease

without undergone oral appliance therapy, Whereas patients of OSA without undergone weight loss or surgical treatment had a higher risk of MACE than patients with OSA with weight loss or surgical treatment, Table S-2. Table S-3 presents the prevalence of type 2 diabetes was higher in OSA patients without underwent weight loss or surgical treatment than OSA patients with weight loss or surgical treatment.

\section{Discussion}

In our cohort study, OSA participants with diabetes had a higher incidence of all end events during the median 
Table 2 Crude number of adverse events during follow-up

\begin{tabular}{llll}
\hline Follow-up outcomes & $\begin{array}{l}\text { Total } \\
(\boldsymbol{n}=\mathbf{1 1 1 3})\end{array}$ & $\begin{array}{l}\text { Diabetes } \\
(\boldsymbol{n}=\mathbf{2 6 6})\end{array}$ & $\begin{array}{l}\text { Non- diabetes } \\
(\boldsymbol{n}=\mathbf{8 4 7})\end{array}$ \\
\hline MACE, $\mathrm{n}(\%)$ & $97(8.7)$ & $33(12.4)$ & $64(7.6)$ \\
Cardiovascular death, $\mathrm{n}(\%)$ & $20(1.8)$ & $6(2.3)$ & $14(1.7)$ \\
Ml, $\mathrm{n}(\%)$ & $26(2.3)$ & $10(3.8)$ & $16(1.9)$ \\
Hospitalization for unstable angina, $\mathrm{n}(\%)$ & $56(5.0)$ & $23(8.6)$ & $33(3.9)$ \\
Hospitalization for heart failure, $\mathrm{n}(\%)$ & $10(0.9)$ & $3(1.1)$ & $7(0.8)$ \\
All-cause mortality, $\mathrm{n}(\%)$ & $43(3.9)$ & $15(5.6)$ & $28(3.3)$ \\
Composite of all events, $\mathrm{n}(\%)$ & $119(10.7)$ & $41(15.5)$ & $78(9.2)$ \\
\hline
\end{tabular}

MACE major adverse cardiovascular event, $M I$ myocardial infarction

42-month follow-up. After adjusting for a range of potential confounders, our study showed a trend of increased risk for MACE, hospitalisation for unstable angina and a composite of all events in OSA patients with type 2 diabetes. Subgroup analysis demonstrated that adjusted hazard ratios for MACE by diabetes were higher in obese and overweight females $\geq 70$ years and patients with mild OSA.

OSA is the most common type of sleep apnoea. It is caused by intermittent upper airway obstruction during sleep, resulting in repeated oxygenated haemoglobin desaturation and sleep fragmentation [26]. Multiple mechanisms link OSA to CVD complications, including insulin resistance, oxidative stress, sympathetic activation, endothelial dysfunction and increased inflammation
[27]. A previous study showed a strong correlation between OSA and cardiac metabolic syndrome. However, this was a retrospective study of young and middle-aged adults [28]. The RICCADSA study also confirmed that OSA was an independent risk factor for poor cardiovascular prognosis in patients with acute coronary syndrome [29]. A meta-analysis revealed that the risk of fatal or non-fatal cardiovascular events in OSA patients was 3 times higher than controls [30]. Another study concluded that the mortality of ST-segment elevation MI in OSA patients was lower than non-OSA patients as OSA could initiate the mechanism of 'ischaemic preconditioning' to protect the myocardium [15]. There is a growing amount of evidence that the evolution of OSA severity is related to a deterioration in blood glucose

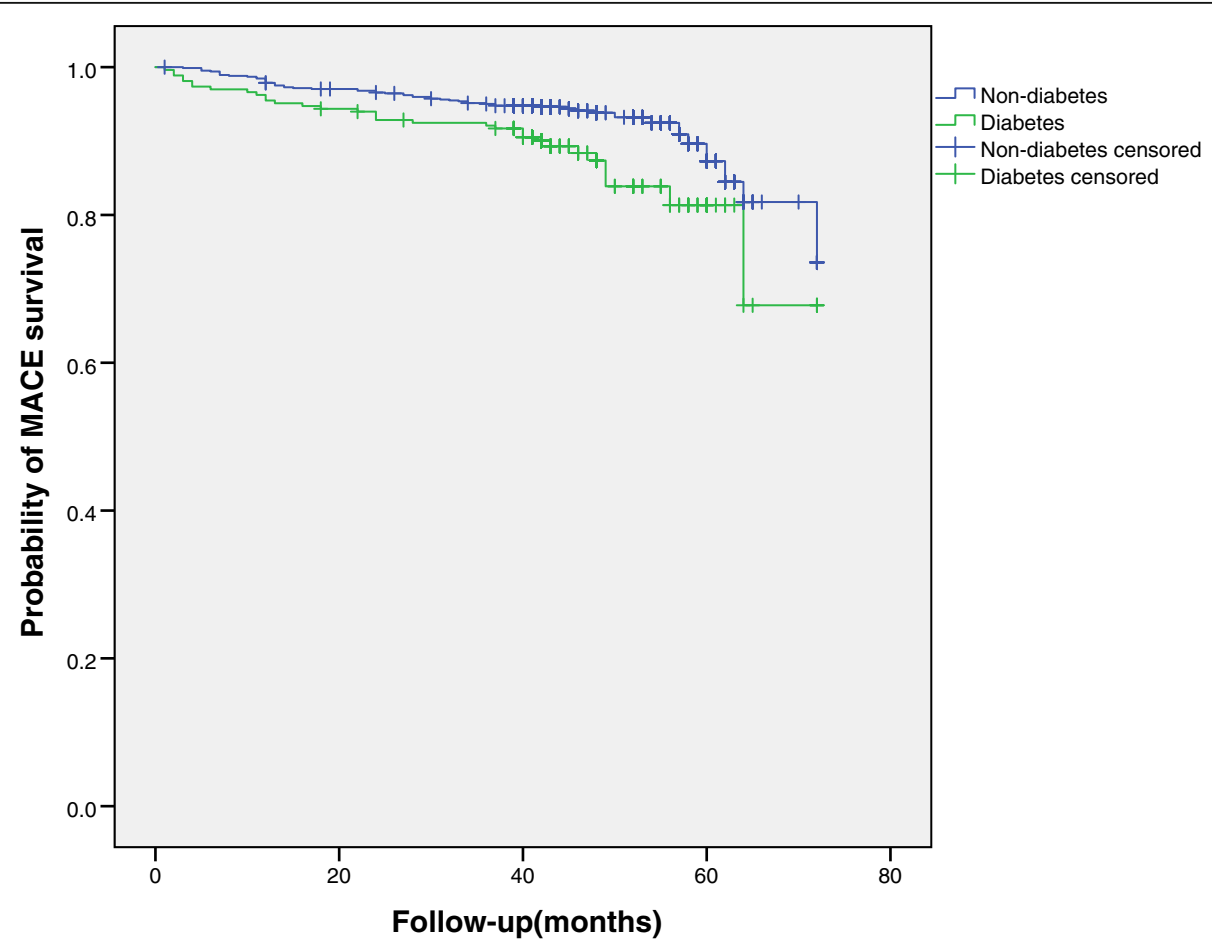

Fig. 2 Kaplan-Meier estimates of probability of survival (\%) for MACE (Primary end point). Log-rank test: $P=0.003$. MACE: major adverse cardiovascular event 
Table 3 Association between type 2 diabetes and incidence of all events

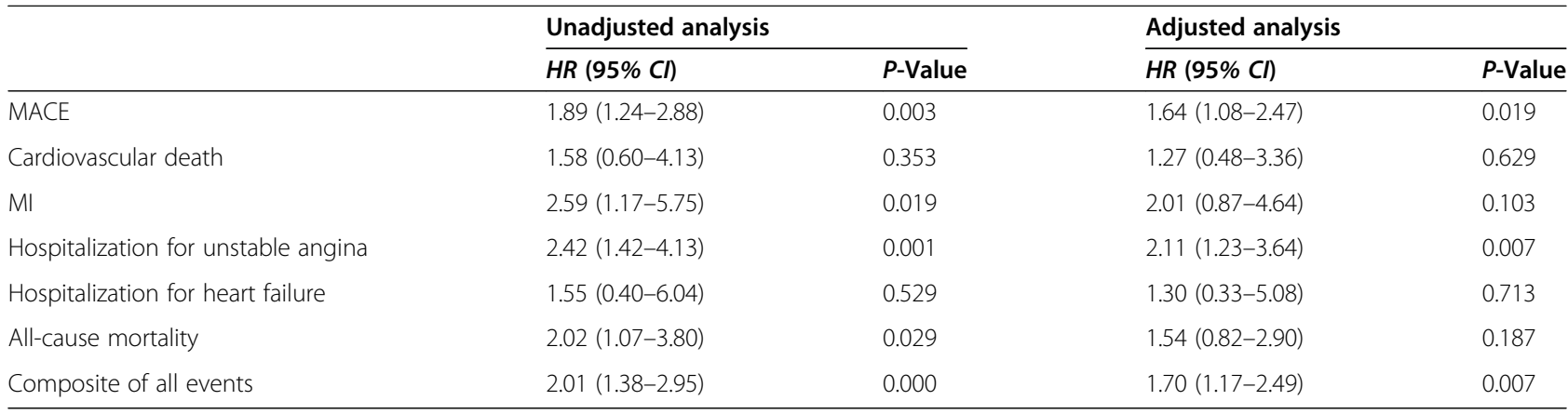

MACE major adverse cardiovascular event, $M I$ myocardial infarction

control [31, 32]. Therefore, our study further investigated the impact of concomitant type 2 diabetes on the long-term risk of MACE in patients with OSA. Notably, our study provided significant findings as a multicentre OSA population-based study adjusted for several potential confounders with confirmed statistical significance for MACE between patients with and without diabetes.

OSA could activate numerous endothelial cells and inflammatory cells and result in endothelial dysfunction, a predictor for MACE [33]. A cohort study proved that severe OSA was associated with cardiovascular events [34]. However, a cross-sectional study confirmed that moderate-severe OSA had no effect on microvascular endothelial function, especially in patients with type 2 diabetes [11]. Statistically speaking, although our data showed no correlation between the evolution of OSA severity and MACE risk in patients with type 2 diabetes, the risk trend for MACE increased in mild OSA patients with type 2 diabetes, which is partly consistent with previous study findings. First, age may be a significant interference factor in the results of this study. Second, severe OSA may involve more effective self-protective mechanisms, such as excessive respiratory effort and/or increased respiratory frequency, compensating for hypoxia in the body to reduce MACE risk.

OSA and all-cause mortality were significantly associated with each other in the general population. A study found that intermittent hypoxia could have protective effects on the cardiovascular system in elderly patients with OSA, reducing the risk of cardiovascular death and all-cause mortality [14]. Our findings showed that type 2 diabetes was nominally associated with the incidence of all-cause mortality and fell short of statistical significance, possibly because $87.6 \%$ of OSA patients with diabetes in our study were in stable condition with no target organ damage. Even so, the potential impact of the complications of diabetes on all-cause mortality and cardiovascular death in OSA patients cannot be ignored, especially in clinical diagnosis and treatment. Edwards et al. demonstrated that the severity of hypoxia caused by OSA in elderly patients is lower than in young patients [35]. Our data showed that the risk of MACE in elderly OSA $\geq 70$ years with concomitant diabetes was

Table 4 Subgroup analysis of the associations between type 2 diabetes and MACE

\begin{tabular}{|c|c|c|c|c|}
\hline & \multicolumn{2}{|c|}{ Unadjusted analysis } & \multicolumn{2}{|c|}{ Adjusted analysis } \\
\hline & $H R(95 \% C l)$ & $P$-Value & HR $(95 \% C l)$ & $P$-Value \\
\hline \multicolumn{5}{|l|}{ Age } \\
\hline$<70$ & $1.62(0.86-3.07)$ & 1.623 & $1.41(0.73-2.69)$ & 0.307 \\
\hline$\geq 70$ & $1.86(1.04-3.32)$ & 0.036 & $1.95(1.08-3.52)$ & 0.027 \\
\hline \multicolumn{5}{|l|}{ Severity of OSA } \\
\hline Mild & $2.48(1.10-5.61)$ & 0.029 & $2.42(1.03-5.71)$ & 0.044 \\
\hline Moderate-severe & $1.76(1.07-2.89)$ & 0.025 & $1.68(0.77-3.65)$ & 0.192 \\
\hline \multicolumn{5}{|l|}{ Gender } \\
\hline Male & $1.71(1.01-2.90)$ & 0.045 & $1.66(0.98-2.81)$ & 0.62 \\
\hline Female & $2.31(1.13-4.70)$ & 0.022 & $2.46(1.17-5.19)$ & 0.018 \\
\hline \multicolumn{5}{|l|}{ BMI } \\
\hline Normal (18.5-22.9) & $1.60(0.46-5.56)$ & 0.463 & $1.90(0.53-6.86)$ & 0.326 \\
\hline Overweight and obese $(\geq 23)$ & $2.02(1.28-3.18)$ & 0.003 & $2.04(1.29-3.33)$ & 0.002 \\
\hline
\end{tabular}




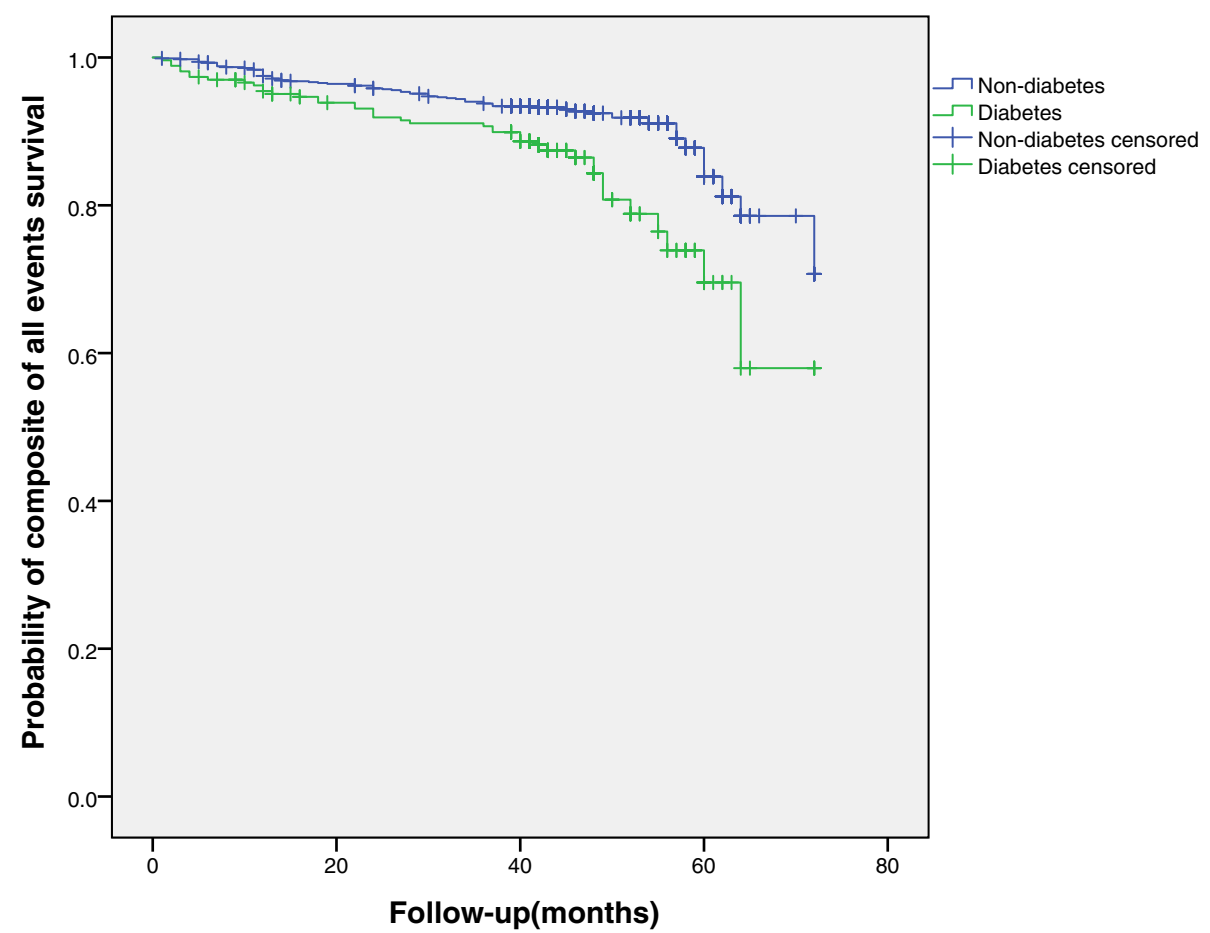

Fig. 3 Kaplan-Meier estimates of probability of survival (\%) for composite of all events. Log-rank test: $P=0.000$

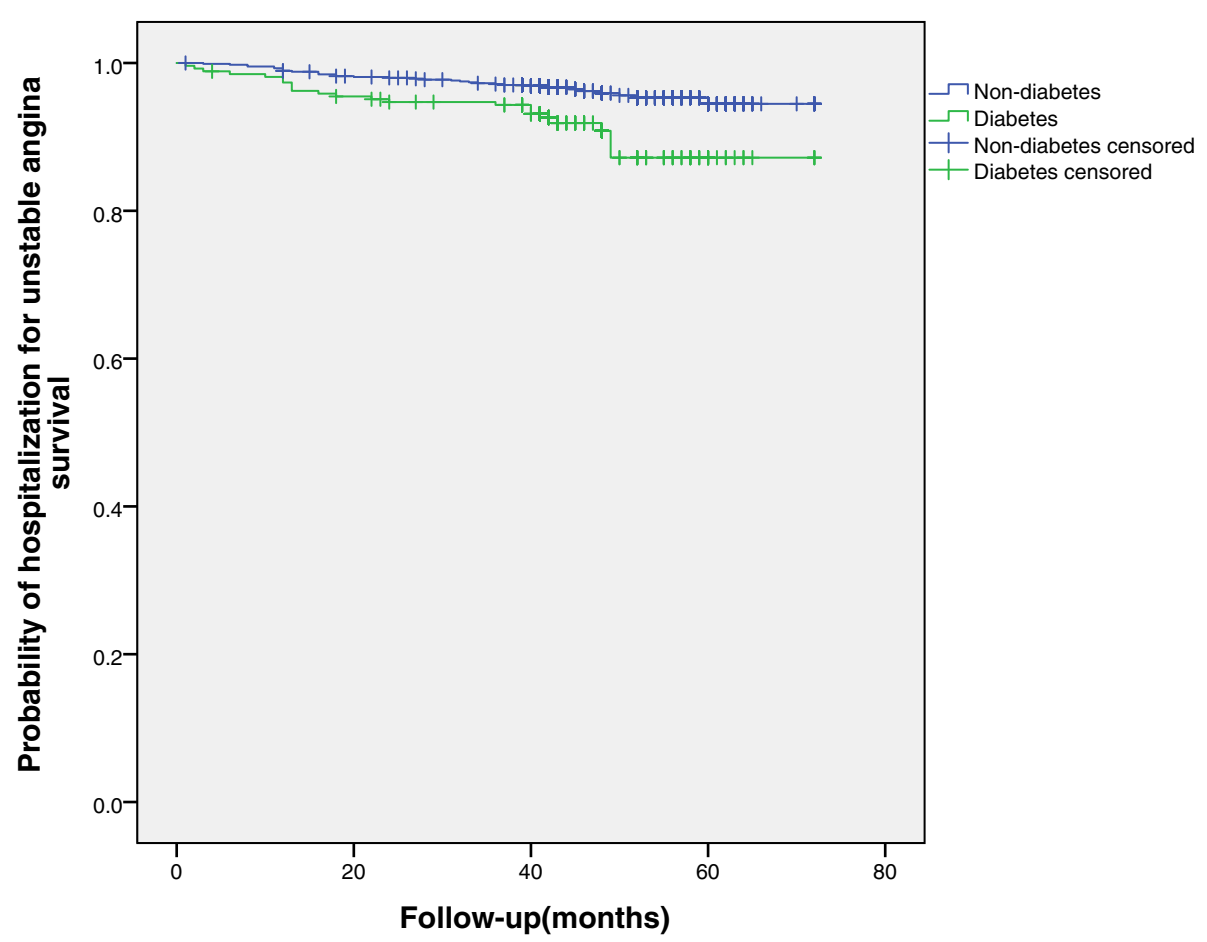

Fig. 4 Kaplan-Meier estimates of probability of survival (\%) for Hospitalization for unstable angina. Log-rank test: $P=0.026$ 
significantly higher than in patients below 70 years, possibly due to the complex symptoms in elderly patients and impaired hypoxia tolerance. This study revealed that type 2 diabetes was associated with a higher risk of MACE in overweight and obese patients with OSA, which is not in line with previous studies. However, the 'obesity paradox phenomenon' indicated that obese patients with cardiovascular disease had a better cardiovascular prognosis than nonobese patients [36]. It is essential to regulate the body mass index, especially in elderly OSA patients with concomitant diabetes.

Evidence reveals that OSA and type 2 diabetes are independent risk factors for cardiovascular disease [1]. Previous studies showed that patients with OSA had a higher risk of cardiovascular disease [37, 38]. However, a prospective survey of an Asian population showed no correlation between OSA and cardiovascular disease [39]. Adderley and Subramanian suggested that prevalent diabetes or incident diabetes during the follow-up period showed a higher CVD risk in OSA patients. However, in this study, most subjects were young and middle-aged patients in the UK [1]. Our study found that the elderly OSA patients with diabetes had a higher risk of MACE, especially females. Therefore, the relationship between diabetes and cardiovascular disease in OSA patients is worthy of further research.

\section{Study limitations}

Our study has several strengths and a few limitations. First, we assessed the risk of CVD and all-cause mortality in the diabetes group and the non-diabetes group of OSA patients without including healthy controls. Second, a median follow-up period of 42 months may be insufficient for all end events development in this cohort. Although this was a multicentre prospective cohort study, the study population consisted of Chinese patients; hence, selection bias could occur. However, these limitations do not affect the value of our study.

\section{Conclusion}

In conclusion, OSA and type 2 diabetes are interrelated and synergistic with MACE, hospitalisation for unstable angina and a composite of all events development. In the subgroup analysis, overweight and obese females, 70 years of age, with mild OSA and concomitant diabetes presented a higher risk of MACE. Physicians need to recognise that patients with OSA complicated with type 2 diabetes constitute a high-risk population requiring strategy implementation to detect type 2 diabetes and prevent vascular complications. Further large-scale cohort studies examining the correlation between OSA, diabetes and cardiovascular disease risk are needed.

\section{Abbreviations}

CVD: Cardiovascular disease; OSA: Obstructive sleep apnoea; MACE: Major adverse cardiovascular events; PSG: Polysomnography; AHI: The apnoeahypopnoea index; BMI: Body mass index; SBP: Systolic blood pressure; DBP: Diastolic blood pressure; ODI: The oxygen desaturation index; MSpO2: The mean pulse oxygen saturation; LSpO2: The lowest pulse oxygen saturation; CHD: Coronary heart disease; COPD: Chronic obstructive pulmonary disease; MI: Myocardial infarction; CPAP: Continuous positive airway pressure; AHR: Adjusted hazard ratios; HR: Hazard ratios;

$\mathrm{Cl}$ : Confidence intervals; HTN: Hypertension

\section{Supplementary Information}

The online version contains supplementary material available at https://doi. org/10.1186/s12877-021-02461-x.

Additional file 1: Supplementary Table s-1. Characterics of covariates. Supplementary Table s-2. Subgroup analysis of the associations between type 2 diabetes and MACE. Supplementary Table s-3. Crude number of type 2 diabetes in treatment for OSA

\section{Acknowledgements}

Not applicable.

\section{Authors' contributions}

$X S, J H L, Y H G, K C, Y G, J G, L Z, H W, M S, X Z, W X, Y W, J L, H X$, JLL and XK collected the data. $X S, J H L$, and $Y H G$ analyzed the data and wrote the manuscript draft. $X Q, J H$ and $L L$ designed this study. All authors have read and approved the manuscript.

\section{Funding}

This study was supported by Military Health Care Project (19BJZ34); Youth Program for Military Medicine of Chinese PLA General Hospital (QNC19054); Open Subject of National Clinical Research Center for Geriatric

Diseases (NCRCG-PLAGH-2018008); Special Project of the Second Medical Center of PLA General Hospital (ZXD2008). The funders had not directly role in the design, data collection, analysis, interpretation or writing of the manuscript.

\section{Availability of data and materials}

Our data may not be shared directly, because it is our teamwork; informed consent should be attained from all the team members. Our data or material may be available after contacting the corresponding author or first author.

\section{Declarations}

Ethics approval and consent to participate

The Ethics Committee of Chinese PLA General Hospital (S2019-352-01) approved the study. Written informed consent was obtained from all participants.

\section{Consent for publication}

Not applicable.

\section{Competing interests}

The authors declare no conflict of interest, financial or otherwise.

\section{Author details}

${ }^{1}$ Medical College, Yan'an University, Yan'an, Shanxi Province, China. ${ }^{2}$ Cardiology Department of the Second Medical Center \& National Clinical Research Center for Geriatric Diseases, Chinese PLA General Hospital, Beijing, China. ${ }^{3}$ PKU-UPenn Sleep Center, Peking University International Hospital, Beijing, China. ${ }^{4}$ Sleep Center, The Affiliated Hospital of Gansu University of Chinese Medicine, Lanzhou City, Gansu Province, China. ${ }^{5}$ Department of General Practice, 960th Hospital of PLA, Jinan, Shandong Province, China. ${ }^{6}$ Sleep Medicine Center, Department of Respiratory and Critical Care Medicine, Peking University People's Hospital, Beijing, China. ${ }^{7}$ Department of Respiratory and Critical Care Medicine of the Second Medical Center \& National Clinical Research Center for Geriatric Diseases, Chinese PLA General Hospital, 28 Fuxing Road, Beijing, China. ${ }^{8}$ Department of Respiratory and 
Critical Care Medicine, Beijing Chaoyang Hospital Affiliated to Capital Medical University, Beijing, China.

\section{Received: 19 June 2021 Accepted: 10 September 2021}

Published online: 25 September 2021

\section{References}

1. Adderley NJ, Subramanian A, Toulis K, et al. Obstructive sleep apnea, a risk factor for cardiovascular and microvascular disease in patients with type 2 diabetes: findings from a population-based cohort study. Diabetes Care. 2020;43(8):1868-77.

2. Mazzotti DR, Keenan BT, Lim DC, Gottlieb DJ, Kim J, Pack Al. Symptom subtypes of obstructive sleep apnea predict incidence of cardiovascular outcomes. Am J Respir Crit Care Med. 2019;200(4):493-506.

3. Chan MTV, Wang CY, Seet E, et al. Association of Unrecognized Obstructive Sleep Apnea with Postoperative Cardiovascular Events in patients undergoing major noncardiac surgery. JAMA. 2019;321(18):1788-98

4. Subramanian A, Adderley NJ, Tracy A, et al. Risk of incident obstructive sleep apnea among patients with type 2 diabetes. Diabetes Care. 2019;42(5): 954-63.

5. Alshehri MA, Alharthi SR, Alsuwat AA, Alswat KA. Clinical characteristics of type 2 diabetics who are at high risk for obstructive sleep apnea. Med Arch. 2018;72(4):249-52.

6. Aurora RN, Punjabi NM. Obstructive sleep apnea, sleepiness, and glycemic control in type 2 diabetes. J Clin Sleep Med. 2019;15(5):749-55.

7. Aurora RN, Punjabi NM. Obstructive sleep apnoea and type 2 diabetes mellitus: a bidirectional association. Lancet Respir Med. 2013;1(4):329-38.

8. Byun Jl, Cha KS, Jun JE, et al. Dynamic changes in nocturnal blood glucose levels are associated with sleep-related features in patients with obstructive sleep apnea. Sci Rep. 2020;10(1):17877.

9. Trinh MD, Plihalova A, Gojda J, et al. Obstructive sleep apnoea increases lipolysis and deteriorates glucose homeostasis in patients with type 2 diabetes mellitus. Sci Rep. 2021;11(1):3567.

10. Fallahi A, Jamil DI, Karimi EB, Baghi V, Gheshlagh RG. Prevalence of obstructive sleep apnea in patients with type 2 diabetes: a systematic review and meta-analysis. Diabetes Metab Syndr. 2019;13(4):2463-8.

11. Bironneau V, Goupil F, Ducluzeau PH, et al. Association between obstructive sleep apnea severity and endothelial dysfunction in patients with type 2 diabetes. Cardiovasc Diabetol. 2017:16(1):39.

12. Chen CL, Liu L, Huang JY, et al. Systolic blood pressure, cardiovascular mortality, and all-cause mortality in Normoglycemia, Prediabetes, and diabetes. Diabetes Metab Syndr Obes. 2020;13:2375-88.

13. Rawshani A, Rawshani A, Franzén S, et al. Risk factors, mortality, and cardiovascular outcomes in patients with type 2 diabetes. N Engl J Med. 2018:379(7):633-44.

14. Lavie P, Lavie L. Unexpected survival advantage in elderly people with moderate sleep apnoea. J Sleep Res. 2009;18(4):397-403.

15. Mohananey D, Villablanca PA, Gupta T, et al. Recognized obstructive sleep apnea is associated with improved in-hospital outcomes after ST elevation myocardial infarction. J Am Heart Assoc. 2017;6(7):e006133.

16. Lavie $P$, Lavie $L$, Herer $P$. All-cause mortality in males with sleep apnoea syndrome: declining mortality rates with age. Eur Respir J. 2005;25(3):514-20.

17. Ogilvie RP, Lakshminarayan K, Iber C, Patel SR, Lutsey PL. Joint effects of OSA and self-reported sleepiness on incident CHD and stroke. Sleep Med. 2018;44:32-7.

18. Qian X, Yin T, Li T, et al. High levels of inflammation and insulin resistance in obstructive sleep apnea patients with hypertension. Inflammation. 2012; 35(4):1507-11.

19. Kapur VK, Auckley DH, Chowdhuri S, et al. Clinical practice guideline for diagnostic testing for adult obstructive sleep apnea: an American Academy of sleep medicine clinical practice guideline. J Clin Sleep Med. 2017;13(3): 479-504.

20. Kendzerska T, Gershon AS, Hawker G, Leung RS, Tomlinson G. Obstructive sleep apnea and risk of cardiovascular events and all-cause mortality: a decade-long historical cohort study. PLoS Med. 2014;11(2):e1001599.

21. Ma YN, Xie WX, Hou ZH, et al. Association between coronary artery calcification and cognitive function in a Chinese community-based population. J Geriatr Cardiol. 2021;18(7):514-22.

22. Joint committee issued Chinese guideline for the management of dyslipidemia in adults. 2016 Chinese guideline for the management of dyslipidemia in adults. Zhonghua Xin Xue Guan Bing Za Zhi. 2016;44(10): 833-53.

23. Kirchhof P, Benussi S, Kotecha D, et al. 2016 ESC guidelines for the management of atrial fibrillation developed in collaboration with EACTS. Eur Heart J. 2016;37(38):2893-962.

24. Charlson ME, Pompei P, Ales KL, MacKenzie CR. A new method of classifying prognostic comorbidity in longitudinal studies: development and validation. J Chronic Dis. 1987;40:373-83.

25. Anno T, Mune T, Takai M, et al. Decreased plasma aldosterone levels in patients with type 2diabetes mellitus: a possible pitfall in diagnosis of primary aldosteronism. Diabetes Metab. 2019;45(4):399-400.

26. Wang G, Goebel JR, Li C, Hallman HG, Gilford TM, Li W. Therapeutic effects of CPAP on cognitive impairments associated with OSA. J Neurol. 2020; 267(10):2823-8

27. Javaheri S, Barbe F, Campos-Rodriguez F, et al. Sleep apnea: types, mechanisms, and clinical cardiovascular consequences. J Am Coll Cardiol. 2017;69(7):841-58.

28. André $\mathrm{S}$, Andreozzi F, Van Overstraeten $\mathrm{C}$, et al. Cardiometabolic comorbidities in obstructive sleep apnea patients are related to disease severity, nocturnal hypoxemia, and decreased sleep quality. Respir Res. 2020;21(1):35.

29. Peker $Y$, Thunström E, Glantz H, Eulenburg C. Effect of obstructive sleep apnea and CPAP treatment on cardiovascular outcomes in acute coronary syndrome in the RICCADSA trial. J Clin Med. 2020;9(12):4051.

30. Peres BU, Hirsch Allen AJ, Fox N, et al. Circulating biomarkers to identify cardiometabolic complications in patients with obstructive sleep apnea: a systematic review. Sleep Med Rev. 2019;44:48-57.

31. Kent BD, Grote L, Bonsignore MR, et al. Sleep apnoea severity independently predicts glycaemic health in nondiabetic subjects: the ESADA study. Eur Respir J. 2014;44(1):130-9.

32. Appleton SL, Vakulin A, Wittert GA, et al. The association of obstructive sleep apnea (OSA) and nocturnal hypoxemia with the development of abnormal $\mathrm{HbA} 1 \mathrm{c}$ in a population cohort of men without diabetes. Diabetes Res Clin Pract. 2016;114:151-9.

33. Shpilsky D, Erqou S, Patel SR, et al. Association of obstructive sleep apnea with microvascular endothelial dysfunction and subclinical coronary artery disease in a community-based population. Vasc Med. 2018;23(4):331-9.

34. Drager LF, Santos RB, Silva WA, et al. OSA, short sleep duration, and their interactions with sleepiness and Cardiometabolic risk factors in adults: the ELSA-Brasil study. Chest. 2019;155(6):1190-8.

35. Edwards BA, Wellman A, Sands SA, et al. Obstructive sleep apnea in older adults is a distinctly different physiological phenotype. Sleep. 2014;37(7): 1227-36.

36. Gupta T, Kolte D, Mohananey D, et al. Relation of obesity to survival after inhospital cardiac arrest. Am J Cardiol. 2016;118(5):662-7.

37. Baumert M, Immanuel SA, Stone $\mathrm{KL}$, et al. Composition of nocturna hypoxaemic burden and its prognostic value for cardiovascular mortality in older community-dwelling men. Eur Heart J. 2020;41(4):533-41.

38. Yaggi HK, Concato J, Kernan WN, Lichtman JH, Brass LM, Mohsenin V. Obstructive sleep apnea as a risk factor for stroke and death. N Engl J Med. 2005;353(19):2034-41.

39. Fan J, Wang X, Ma X, Somers VK, Nie S, Wei Y. Association of Obstructive Sleep Apnea with Cardiovascular Outcomes in patients with acute coronary syndrome. J Am Heart Assoc. 2019;8(2):e010826.

\section{Publisher's Note}

Springer Nature remains neutral with regard to jurisdictional claims in published maps and institutional affiliations. 